\title{
Inexact SA Method for Constrained Stochastic Convex SDP and Application in Chinese Stock Market
}

\author{
Shuang Chen $\mathbb{D}^{1},{ }^{1}$ Li-Ping Pang, ${ }^{2}$ Jian $\mathrm{Lv}^{3}$ and Zun-Quan Xia ${ }^{2}$ \\ ${ }^{1}$ Information and Engineering College, Dalian University, Dalian, China \\ ${ }^{2}$ School of Mathematical Sciences, Dalian University of Technology, Dalian, China \\ ${ }^{3}$ School of Finance, Zhejiang University of Finance and Economics, Hangzhou, China \\ Correspondence should be addressed to Shuang Chen; chenshuang0707@163.com
}

Received 4 August 2017; Revised 17 November 2017; Accepted 13 December 2017; Published 23 January 2018

Academic Editor: Dhananjay Gopal

Copyright (C) 2018 Shuang Chen et al. This is an open access article distributed under the Creative Commons Attribution License, which permits unrestricted use, distribution, and reproduction in any medium, provided the original work is properly cited.

We propose stochastic convex semidefinite programs (SCSDPs) to handle uncertain data in applications. For these models, we design an efficient inexact stochastic approximation (SA) method and prove the convergence, complexity, and robust treatment of the algorithm. We apply the inexact method for solving SCSDPs where the subproblem in each iteration is only solved approximately and show that it enjoys the similar iteration complexity as the exact counterpart if the subproblems are progressively solved to sufficient accuracy. Numerical experiments show that the method we proposed was effective for uncertain problem.

\section{Introduction}

In this paper, we propose a class of optimization problems called stochastic convex semidefinite programs (SCSDPs):

$$
\begin{array}{cl}
\min & f(X)=\mathbb{E}[F(X, \xi)] \\
\text { s.t. } & \mathscr{A}(X)=b, \\
& X \geq 0, X \in S^{n},
\end{array}
$$

where $F$ is a smooth convex function for every realization of $\xi$ on $S_{+}^{n}$, $\xi$ is a random matrix whose probability distribution $P$ is supported on set $\Omega \in R^{r \times r}, \mathscr{A}: S^{n} \rightarrow R^{m}$ is a linear map, $b \in R^{m}$ and $X \geq 0$ mean that $X \in S_{+}^{n}$, and $S^{n}$ is the space of $n \times n$ real symmetric endowed with the standard trace inner product $\langle\cdot, \cdot\rangle$ and Frobenius norm $\|\cdot\|_{F} . S_{+}^{n}$ is the set of positive semidefinite matrices in $S^{n}$.

SCSDPs may be viewed as an extension of the following stochastic models:

(1) Stochastic (Linear) Semidefinite Programs (SLSDPs) ([1-5])

$$
\begin{array}{ll}
\min & \langle C, X\rangle+E[Q(X, w)] \\
\text { s.t. } & \left\langle A_{i}, X\right\rangle=b_{i}, \quad i=1, \ldots, m_{1}, \\
& X \geq 0,
\end{array}
$$

where $Q(X, w)$ is the minimum of the problem

$$
\begin{array}{ll}
\min & \langle D(w), Y\rangle \\
\text { s.t. } & \left\langle T_{j}, X\right\rangle+\left\langle W_{j}, Y\right\rangle=d_{j}(w), \quad i=1, \ldots, m_{2}, \\
& Y \geq 0,
\end{array}
$$

where $\langle A, B\rangle=\operatorname{trace}\left(A^{T} B\right)$ denotes the Frobenius inner product between $A$ and $B$.

\section{(2) Stochastic Convex Quadratic Semidefinite Programs} (SCQSDPs)

$$
\begin{array}{ll}
\min & \frac{1}{2}\langle X, \mathbb{E}[Q(X, \xi)]\rangle+\langle C, X\rangle \\
\text { s.t. } & \mathscr{A}(X)=b, \\
& X \geq 0,
\end{array}
$$

where $Q(\cdot, \xi): S^{n} \times \Omega \rightarrow S^{n}$ is given self-adjoint positive semidefinite linear operator for every $\xi \in \Omega$ and $C \in S^{n}$.

(3) Stochastic Nearest Correlation Matrix. Now we consider an interesting example from finance industry. In stock research, sample correlation matrices (a symmetric positive semidefinite matrix with unit diagonal) constructed from vectors of 
stock returns are used for predictive purposes. Unfortunately, on any day when an observation is made data is rarely available for all the stocks of interest. Higham [6] proposed a method that is to compute the sample correlations of pairs of stocks using data drawn only from the days on which both stocks have data available. Compute the nearest correlation matrix and use it for the subsequent stock analysis. This is a statistical application that motivates nearest correlation matrix problem

$$
\begin{array}{ll}
\min & \frac{1}{2}\|\mathscr{L}(X-U)\|^{2} \\
\text { s.t. } & \operatorname{diag}(X)=e \\
& X \geq 0,
\end{array}
$$

where $e \in R^{n}$ is a vector of all ones and $U \in S^{n}$ is given.

Furthermore, with the particularity of Chinese stock market (in Chinese stock market which is different from stock markets in other countries, stock price rise or fall during a day does not exceed ten percent), we could more accurately estimate the information of stock price in the future. We consider computing expected correlations of pairs of stocks returns for better response random factors of the stock market. In order to justify the subsequent stock analysis, it is desired to compute the nearest expectation correlation matrix and to use that matrix in the computation. We use this matrix to predict the correlation of these stocks in the future. The problem we consider is an important special case of (4)

$$
\begin{array}{ll}
\min & \frac{1}{2} E\left[\|\mathscr{L}(X-U(\xi))\|^{2}\right] \\
\text { s.t. } & \operatorname{diag}(X)=e \\
& X \geq 0,
\end{array}
$$

where $U(\xi)$ is a stochastic matrix from $(\mathscr{F}, \Omega, P) \rightarrow S^{n}$.

Alternatively, SCSDPs may be viewed as an extension of the following deterministic convex semidefinite programs $([7-11])$ :

$$
\begin{array}{ll}
\min & F(X) \\
\text { s.t. } & \mathscr{A}(X)=b, \\
& X \geq 0, X \in S^{n},
\end{array}
$$

where $F$ is a smooth convex function on $S_{+}^{n}$ and $\mathscr{A}: S^{n} \rightarrow R^{m}$ is a linear map and $b \in R^{m}$ and $X \geq 0$ mean that $X \in S_{+}^{n}$.

There are several methods available for solving determinate deterministic convex semidefinite programs and their special cases, which include the accelerated proximal gradient method [8], the alternating projection method [6], the quasiNewton method [12], the inexact semismooth Newton-CG method [13], and the inexact interior-point method [14]. However, all the methods mentioned above may not be extended to efficiently solve the SCSDPs (1) because it might not be easy to evaluate $E[f(X, \xi)]$ in the following occasions:

(a) $\xi$ is a random vector with a known probability distribution, but calculations of the expected value involve multidimensional integration, which is computationally expensive if not impossible.

(b) The function $f(X, \xi)$ is known, but the distribution of $\xi$ is unknown and the information on $\xi$ can only be obtained using past data or sampling.

(c) $E[f(X, \xi)]$ is not observable and it must be approximately evaluated through simulation.

Under these circumstances, the existing numerical methods for deterministic convex semidefinite programs are not applicable to SCSDPs and new methods are needed. On the other hand, Ariyawansa and Zhu [1] and Mehrotra and Gozevin [4] apply barrier decomposition algorithms and interior-point methods for solving stochastic (linear) semidefinite programs. However, these methods may not be extended for solving the convex problems.

The main purpose of this paper is to design an efficient algorithm to solve the general problems (1) including all the special cases mentioned above. The algorithm we propose here is based on the classical SA algorithm whose subproblem must be solved exactly to generate the next iterate point. The SA algorithm approach originates from the pioneering work of Robbins and Monro and is discussed in numerous publications [15-18]. In this paper, we design an inexact SA method which overcomes the limitation just mentioned. Specifically, in our inexact SA method, the subproblem is only solved approximately and we allow the error in solving subproblem to be deterministic or stochastic. From the theoretical point of view we analyze the convergence, complexity, and robust treatment of the algorithm. We also give the numerical results for illustrating the effectiveness of our method.

The rest of the paper is organized as follows. In Section 2, we focus on theory of the inexact SA method to solve the more general model-stochastic convex matrix program over abstract set $\Theta$, and the proof of convergence is given whether the error is determinate or stochastic. In Section 3, we provide two error estimations of the algorithm. In Section 4, we apply the theoretical results proved before for solving SCSDPs (1). Numerical experiments will be showed at last.

\section{Algorithm and Convergence Analysis of General Convex Stochastic Matrix Optimization}

For more generality, we first consider the following stochastic convex optimization problem:

$$
\min _{X \in \Theta}\{f(X)=\mathbb{E}[F(X, \xi)]\},
$$

where $\Theta \subset R^{n \times n}$ is a nonempty closed convex set, $\xi$ is a random matrix whose probability distribution $P$ is supported on set $\Omega \subset R^{n \times n}$, and $F$ is a smooth convex stochastic function on $\Theta \times \Omega$. We assume that the expectation

$$
\mathbb{E}[F(X, \xi)]=\int_{\Omega} F(X, \xi) d P(\xi)
$$

is well defined and finite valued for every $X \in \Theta$. We also assume that the expected value function $f(\cdot)$ is continuous 
and convex on $\Theta$. If for every $\xi \in \Omega$ the function $F(\cdot, \xi)$ is convex on $X$, then it follows that $f(\cdot)$ is convex. With these assumptions, (8) becomes a convex programming problem.

Let $f$ be any convex finite at $\Theta$. A matrix $X^{*}$ is called $\varepsilon$ subgradient of $f$ at $X$ (where $\varepsilon>0$ ) if

$$
f(X) \geq(f(X)-\varepsilon)+\left\langle X^{*}, Z-X\right\rangle, \quad \forall Z \in \Theta .
$$

The set of all such $\varepsilon$-subgradients is denoted by $\partial_{\varepsilon} f(X)$.

Next we make the following assumptions (also in [19-21]) which will be used to analyze the convergence of algorithms in this paper:

(A1) It is possible to generate an independent identically distributed i.i.d sample $\xi_{1}, \xi_{2}, \ldots$ of realizations of random vector $\xi$.

(A2) There is an oracle, which, for a given input point $(X, \xi) \in \Theta \times \Omega$, returns inexact stochastic subgradienta vector $G(X, \xi)$ such that $g(X)=\mathbb{E}[G(X, \xi)]$ is well defined and is a $\varepsilon$-subgradient of $f(\cdot)$ at $X$, that is, $g(X) \in \partial_{\varepsilon} f(X)$.

(A3) There is a positive number $M$ such that

$$
\mathbb{E}\left[\|G(X, \xi)\|_{F}^{2}\right] \leq M^{2}, \quad \forall X \in \Theta .
$$

Remark 1. Assumption (A2) is different from the assumption used in [19]. We only need to get a $\varepsilon$-subgradient which is more likely got in practice.

Definition 2. The convex hull of set $S$ (denoted by $\operatorname{co}(S)$ ) is the smallest convex polygon that contains all the points of $S$.

Throughout the paper, we use the following notations. $\|X\|_{F}=\sqrt{\sum_{i, j} X_{i j}^{2}}$ denotes the Frobenius norm, where $X_{i j}^{2}$ means the element at the $i$ th row and $j$ th column of $X$. $\langle X, Y\rangle=\operatorname{Trace}\left(X^{T} Y\right)$ denotes standard trace inner product. By $\Pi_{\Theta}$, we denote the metric projection operator onto the set $X$ : that is, $\Pi_{\Theta}(X)=\arg \min _{X^{\prime} \in \Theta}\left\|X-X^{\prime}\right\|_{F}$. Note that $\Pi_{\Theta}$ is a nonexpanding operator, that is,

$$
\left\|\Pi_{\Theta^{\prime}}-\Pi_{\Theta}\right\|_{F} \leq\left\|X^{\prime}-X\right\|_{F}, \quad \forall X, X^{\prime} \in R^{n} .
$$

The notation $[a]$ stands for the largest integer less than or equal to $a \in R$. By $\xi_{[t]}=\left(\xi_{1}, \ldots, \xi_{t}\right)$, we denote the history of the process $\xi_{1}, \ldots, \xi_{t}$ up to time $t$. Unless stated otherwise, all relations between random variables are supposed to hold almost surely.

In the following, we discuss theory of the inexact SA approach to the minimization problem (8).

The inexact SA algorithm solves (8) by mimicking the subgradient descent method

$$
X_{j+1}=\Pi_{\Theta}\left(X_{j}-\gamma_{j} f^{\prime}\left(X_{j}\right)\right) \text {, }
$$

where $f^{\prime}(X) \in \partial f(X)$. These lead to an executable algorithm below.
Algorithm 3.

Step 1. Give the initial point $X_{1} \in \Theta$ and set $j=1$, iterate the following steps.

Step 2. Choose suitable step sizes $\gamma_{j}>0$ and computational error $e_{j}$.

Step 3. Find an approximation solution of

$$
X_{j+1}=\Pi_{\Theta}\left(X_{j}-\gamma_{j} G\left(X_{j}, \xi_{j}\right)-\gamma_{j} e_{j}\right) .
$$

Set $j:=j+1$; go back to Step 2 .

In order to prove the convergence of Algorithm 3 we introduce the following lemma.

Lemma 4 (see [22]). Let $\mathscr{F}_{k}$ be an increasing sequence of $\sigma$-algebras and let $\left\{t_{k}\right\},\left\{\alpha_{k}\right\},\left\{\beta_{k}\right\}$, and $\left\{\gamma_{k}\right\}$ be nonnegative random variables adapted to $\mathscr{F}_{k}$. If $\Sigma_{i=1}^{\infty} \alpha_{k}<\infty, \Sigma_{i=1}^{\infty} \beta_{k}<\infty$, it holds almost surely that

$$
t_{k+1} \leq\left(1+\alpha_{k}\right) t_{k}+\beta_{k}-\rho_{k}
$$

and then $t_{k}$ is convergent almost surely and $\Sigma \rho_{k}<\infty$ almost surely.

We now state the main convergence theorem.

Theorem 5. Suppose that the stochastic optimization problem (8) has an optimal solution $X^{*}$ and $e_{j} \rightarrow 0$. Let $\gamma_{j}=\theta / j$, where $\theta>0$, and $X_{j} \subset \Theta$ be a sequence generated by (14); then $X_{j}$ converges to $X^{*}$.

Proof. Note that the iterate $X_{j}=X_{j}\left(\xi_{[j-1]}\right)$ is a function of the history $\xi_{[j-1]}=\left(\xi_{1}, \ldots, \xi_{j-1}\right)$ of the generated random process and hence is random.

Denote

$$
\begin{aligned}
D_{j} & =\frac{1}{2}\left\|X_{j}-X^{*}\right\|_{F}^{2}, \\
d_{j} & =\mathbb{E}\left[D_{j}\right]=\frac{1}{2} \mathbb{E}\left[\left\|X_{j}-X^{*}\right\|_{F}^{2}\right] .
\end{aligned}
$$

Since $X^{*} \in \Theta$ and hence $\Pi_{\Theta}\left(X^{*}\right)=X^{*}$, we can write

$$
\begin{aligned}
D_{j+1} & =\frac{1}{2}\left\|\Pi_{\Theta}\left(X_{j}-\gamma_{j} G\left(X_{j}, \xi_{j}\right)-\gamma_{j} e_{j}\right)-X^{*}\right\|_{F}^{2} \\
= & \frac{1}{2}\left\|\Pi_{\Theta}\left(X_{j}-\gamma_{j} G\left(X_{j}, \xi_{j}\right)-\gamma_{j} e_{j}\right)-\Pi_{\Theta}\left(X^{*}\right)\right\|_{F}^{2} \\
\leq & \frac{1}{2}\left\|X_{j}-\gamma_{j} G\left(X_{j}, \xi_{j}\right)-\gamma_{j} e_{j}-X^{*}\right\|_{F}^{2} \\
= & D_{j}+\frac{1}{2} \gamma_{j}^{2}\left\|G\left(X_{j}, \xi_{j}\right)+e_{j}\right\|_{F}^{2} \\
& -\gamma_{j}\left\langle X_{j}-X^{*}, G\left(X_{j}, \xi_{j}\right)+e_{j}\right\rangle,
\end{aligned}
$$

where the second inequality is due to nonexpansionary of projection operator. 
Since $X_{j}$ is independent of $\xi_{j}$, we have

$$
\begin{aligned}
\mathbb{E} & {\left[\left\langle X_{j}-X^{*}, G\left(X_{j}, \xi_{j}\right)\right\rangle\right] } \\
& =\mathbb{E}\left\{\mathbb{E}\left[\left\langle X_{j}-X^{*}, G\left(X_{j}, \xi_{j}\right)\right\rangle \mid \xi_{[j-1]}\right]\right\} \\
& =\mathbb{E}\left\{\left\langle X_{j}-X^{*}, \mathbb{E}\left[G\left(X_{j}, \xi_{j}\right) \mid \xi_{[j-1]}\right]\right\rangle\right\} \\
& =\mathbb{E}\left[\left\langle X_{j}-X^{*}, g\left(X_{j}\right)\right\rangle\right] .
\end{aligned}
$$

Since assumption (A3) and $e_{j} \rightarrow 0$, there is a positive number $M$ such that

$$
\begin{aligned}
D_{j+1} \leq & D_{j}+\frac{1}{2} \gamma_{j}^{2} M^{2} \\
& -\gamma_{j}\left\langle X_{j}-X^{*}, G\left(X_{j}, \xi_{j}\right)+e_{j}\right\rangle .
\end{aligned}
$$

Then, by taking expectation of both sides of (17) and using (18), we obtain

$$
d_{j+1} \leq d_{j}-\gamma_{j} \mathbb{E}\left[\left\langle X_{j}-X^{*}, g\left(X_{j}\right)+e_{j}\right\rangle\right]+\frac{1}{2} \gamma_{j}^{2} M^{2} .
$$

For $\gamma_{j}=\theta / j$, we known that $\sum \theta^{2} / j^{2}<\infty$. By Lemma 4 , we know that $d_{j}=(1 / 2) \mathbb{E}\left[\left\|X_{j}-X^{*}\right\|_{F}^{2}\right]$ is convergent almost surely and

$$
\sum_{j=1}^{\infty} \gamma_{j} \mathbb{E}\left[\left\langle X_{j}-X^{*}, g\left(X_{j}\right)+e_{j}\right\rangle\right]<\infty
$$

Suppose that $X_{j} \rightarrow \bar{X} \neq X^{*}$ and by the convexity of $f(x)$, we have that $\mathbb{E}\left[\left\langle X_{j}-X^{*}, g\left(X_{j}\right)\right\rangle\right] \geq \mathbb{E}\left[f\left(x_{j}\right)-f\left(X^{*}\right)\right]>0$, when $j \rightarrow \infty$. For $\sum_{j=1}^{\infty} \gamma_{j}=\sum_{j=1}^{\infty} \theta / j=\infty$, we know that

$$
\sum_{j=1}^{\infty} \gamma_{j} \mathbb{E}\left[\left\langle X_{j}-X^{*}, g\left(X_{j}\right)+e_{j}\right\rangle\right]=\infty
$$

It is in contradiction with (21); then we have $\mathbb{E}\left[\| X_{j}-\right.$ $\left.X^{*} \|^{2}\right] \rightarrow 0$ almost surely.

Sometimes, the error at each iteration $j$ is relevant to the random variable $\xi_{j}$. In this environment, we consider stochastic inexact SA algorithm.

\section{Algorithm 6.}

Step 1. Give the initial point $X_{1} \in \Theta$ and set $k=1$; iterate the following steps.

Step 2. Choose suitable step sizes $\gamma_{j}>0$ and computational stochastic error $e_{j}\left(\xi_{j}\right)$.

Step 3. Find an approximation solution of

$$
X_{j+1}=\Pi_{\Theta}\left(X_{j}-\gamma_{j} G\left(X_{j}, \xi_{j}\right)-\gamma_{j} e_{j}\left(\xi_{j}\right)\right) \text {. }
$$

Set $j:=j+1$; go back to Step 2 .
Theorem 7. Suppose that the stochastic optimization problem (8) has an optimal solution $X^{*}$. We also supposed that stochastic error $e_{j}\left(\xi_{j}\right) \rightarrow 0$ and there is a positive number $N$ such that $e_{j}\left(\xi_{j}\right) \leq N$. Let $\gamma_{j}=\theta / j$, where $\theta>0$, and $X_{j} \subset \Theta$ be a sequence generated by (23); then $X_{j}$ converges to $X^{*}$.

Proof. Like the proof of Theorem 5, we obtain

$$
\begin{aligned}
d_{j+1} \leq & d_{j}-\gamma_{j} \mathbb{E}\left[\left\langle X_{j}-X^{*}, g\left(X_{j}\right)+\mathbb{E}\left[e_{j}\left(\xi_{j}\right)\right]\right\rangle\right] \\
& +\frac{1}{2} \gamma_{j}^{2}(M+N)^{2} .
\end{aligned}
$$

For $\gamma_{j}=\theta / j$, we known that $\sum \theta^{2} / j^{2}<\infty$. By Lemma 4, we know that $d_{j}=(1 / 2) \mathbb{E}\left[\left\|X_{j}-X^{*}\right\|_{F}^{2}\right]$ is convergent almost surely and

$$
\sum_{j=1}^{\infty} \gamma_{j} \mathbb{E}\left[\left\langle X_{j}-X^{*}, g\left(X_{j}\right)+\mathbb{E}\left[e_{j}\left(\xi_{j}\right)\right]\right\rangle\right]<\infty .
$$

Suppose that $X_{j} \rightarrow \bar{X} \neq X^{*}$ and by the convexity of $f(x)$, we have that $\mathbb{E}\left[\left\langle X_{j}-X^{*}, g\left(X_{j}\right)\right\rangle\right] \geq \mathbb{E}\left[f\left(x_{j}\right)-f\left(X^{*}\right)\right]>0$, when $j \rightarrow \infty$. For $\sum_{j=1}^{\infty} \gamma_{j}=\sum_{j=1}^{\infty} \theta / j=\infty$, we know that

$$
\sum_{j=1}^{\infty} \gamma_{j} \mathbb{E}\left[\left\langle X_{j}-X^{*}, g\left(X_{j}\right)+E\left[e_{j}\left(\xi_{j}\right)\right]\right\rangle\right]=\infty .
$$

It is in contradiction with (25); then we have $\mathbb{E}\left[\| X_{j}-\right.$ $\left.X^{*} \|_{F}\right] \rightarrow 0$ almost surely.

\section{Error Estimation}

Suppose further that the expectation function $f(X)$ is differentiable and strongly convex on $\Theta$; that is, there is constant $c>0$ such that

$$
\begin{aligned}
&\left\langle X^{\prime}-X, \nabla f\left(X^{\prime}\right)-\nabla f(X)\right\rangle \geq c\left\|X^{\prime}-X\right\|_{F}^{2}, \\
& \forall X^{\prime}, X \in \Theta .
\end{aligned}
$$

Next we will discuss the complexity of the above algorithms; we take the second algorithm, for instance.

Theorem 8. Suppose that $f(X)$ is differentiable and strongly convex, $\nabla f(X)$ is Lipschitz continuous, and the conditions of Theorem 7 are satisfied; we have that

$$
\mathbb{E}\left[f\left(X_{j}\right)-f\left(X^{*}\right)\right] \leq \frac{(1 / 2) L Q(\theta)}{j},
$$

where

$$
\begin{aligned}
& Q(\theta) \\
& \quad=\max \left\{\theta^{2}(M+N)^{2}(2 c \theta-1)^{-1},\left\|X_{1}-X^{*}\right\|_{F}^{2}\right\} .
\end{aligned}
$$

Proof. Note that strong convexity of $f(X)$ implies that the minimizer $X^{*}$ is unique. As optimality of $X^{*}$ is unique, we have that

$$
\left\langle X-X^{*}, \nabla f\left(X^{*}\right)\right\rangle \geq 0 \quad \forall X \in \Theta
$$


which together with (27) implies that $\left\langle X-X^{*}, \nabla f(X)\right\rangle \geq$ $c\left\|X^{\prime}-X_{*}\right\|_{F}^{2}$. In turn, it follows that $\left\langle X-X^{*}, g+\mathbb{E}\left[e_{j}\left(\xi_{j}\right)\right]\right\rangle \geq$ $c\left\|X^{\prime}-X_{*}\right\|_{F}^{2}$ for all $X \in \Theta$ and $g\left(X_{j}\right)+\mathbb{E}\left[e_{j}\left(\xi_{j}\right)\right] \in \partial f\left(X_{j}\right)$ and hence

$$
\begin{gathered}
\mathbb{E}\left[\left\langle X_{j}-X^{*}, g\left(X_{j}\right)+\mathbb{E}\left[e_{j}\left(\xi_{j}\right)\right]\right\rangle\right] \\
\geq c \mathbb{E}\left[\left\|X_{j}-X^{*}\right\|_{F}^{2}\right]=2 c d_{j} .
\end{gathered}
$$

Therefore, it follows from (24) that

$$
d_{j+1} \leq d_{j}\left(1-2 c \gamma_{j}\right)+\frac{1}{2} \gamma_{j}^{2}(M+N)^{2} .
$$

For some constant $\theta>1 /(2 c)$, by (32), we have

$$
d_{j+1} \leq d_{j}\left(1-\frac{2 c \theta}{j}\right)+\frac{(1 / 2) \theta^{2}}{j^{2}(M+N)^{2}} .
$$

Next we will prove that

$$
\mathbb{E}\left[\left\|X_{j}-X^{*}\right\|_{F}^{2}\right]=2 d_{j} \leq \frac{Q(\theta)}{j},
$$

where

$$
\begin{aligned}
& Q(\theta) \\
& \quad=\max \left\{\theta^{2}(M+N)^{2}(2 c \theta-1)^{-1},\left\|X_{1}-X^{*}\right\|_{F}^{2}\right\} .
\end{aligned}
$$

Using mathematical induction, for $j=1$, we have

$$
\mathbb{E}\left[\left\|X_{1}-X^{*}\right\|_{F}^{2}\right]=2 d_{1} \leq Q(\theta),
$$

which holds naturally. For

$$
\begin{aligned}
& 2 d_{j+1} \leq \frac{Q(\theta)}{j}\left(1-2 c \frac{\theta}{j}\right)+\frac{\theta^{2}}{j^{2}}(M+N)^{2} \\
& =\frac{\theta^{2}(M+N)^{2}(j-2 c \theta)+\theta^{2}(M+N)^{2}(2 c \theta-1)}{j^{2}(2 c \theta-1)} \\
& \quad=\frac{\theta^{2}(M+N)^{2}(j-1)}{j^{2}(2 c \theta-1)}, \\
& \frac{Q(\theta)}{j+1}=\frac{\theta^{2}(M+N)^{2}}{(2 c \theta-1)(j+1)},
\end{aligned}
$$

we know that $(*) /(* *)<1$; then we have $\mathbb{E}\left[\left\|X_{j}-X^{*}\right\|_{F}\right]=$ $2 d_{j} \leq Q(\theta) / j$.

For $\nabla f(X)$ is Lipschitz continuous, there is constant $L>0$ such that

$$
\left\|\nabla f\left(X^{\prime}\right)-\nabla f(X)\right\|_{F} \leq L\left\|X^{\prime}-X\right\|_{F}, \quad \forall X^{\prime}, X \in \Theta,
$$

and hence

$$
\begin{aligned}
\mathbb{E} & {\left[f\left(X_{j}\right)-f\left(X^{*}\right)\right] } \\
& \leq \mathbb{E}\left[-\nabla f\left(X_{j}\right)\left(X_{*}-X_{j}\right)+\nabla f\left(X^{*}\right)\left(X_{*}-X_{j}\right)\right]
\end{aligned}
$$

$$
\begin{aligned}
& =\mathbb{E}\left[\left(\nabla f\left(X^{*}\right)-\nabla f\left(X_{j}\right)\right)\left(X_{*}-X_{j}\right)\right] \\
& \leq L \mathbb{E}\left[\left\|X_{j}-X^{*}\right\|_{F}^{2}\right] \leq \frac{L Q(\theta)}{j} .
\end{aligned}
$$

When $\theta>1 / 2 c$, it follows from (38) that, after $j$ iterations, the expected error in terms of the objective value is of order $O\left(j^{-1}\right)$. But that the result is highly sensitive to a priori information on strongly convex factor $c$. In order to make the SA method applicable to general convex objectives rather than to strongly convex ones, one should replace the classical step sizes $\gamma_{j}=\theta / j$, which can be too small to ensure a reasonable rate of convergence. We take appropriate averages of the search points $X_{j}$ rather than these points themselves. This processing method go back to $[20,23]$ and we call it "robust treatment." We will show the effectiveness of the above technique in the following theorem.

Theorem 9. Suppose that $f$ is convex; let $v_{t} \geq 0$ and $\sum_{t=i}^{j} v_{t}=$ 1. Consider the points $\widetilde{X}_{i}^{j}=\sum_{t=i}^{j} v_{t} X_{t}$, and let

$$
D_{\Theta}=\max _{X \in \operatorname{co}\left\{X_{k}\right\}}\left\|X_{k}-X_{1}\right\|_{F} .
$$

We know that

$$
\mathbb{E}\left[f\left(\widetilde{X}_{i}^{j}\right)-f\left(X^{*}\right)\right] \leq \frac{D_{\Theta}^{2}(5(M+N)+\sqrt{N})}{2 n \gamma} .
$$

Proof. Due to (24) and assumption (A3), we know that

$$
\begin{array}{rl}
\gamma_{t} & \mathbb{E} \\
& \left.\left(f\left(X_{t}\right)-f\left(X^{*}\right)\right)\right] \leq \gamma_{t}\left\langle X_{t}-X^{*}, G\left(X_{t}, \xi_{j}\right)\right\rangle \\
\leq & d_{t}-d_{t+1}+\frac{1}{2} \gamma_{t}^{2}(M+N)^{2} \\
& -\gamma_{t}\left\langle\mathbb{E}\left[X_{t}-X^{*}\right], \mathbb{E}\left[e_{t}\left(\xi_{t}\right)\right]\right\rangle .
\end{array}
$$

It follows that whenever $1 \leq i \leq j$, we have

$$
\begin{array}{rl}
\sum_{t=i}^{j} \gamma_{t} & \mathbb{E} \\
\leq & \left.f\left(X_{t}\right)-f\left(X^{*}\right)\right] \\
& \sum_{t=i}^{j}\left[d_{t}-d_{t+1}\right]+\frac{1}{2}(M+N)^{2} \sum_{t=i}^{j} \gamma_{t}^{2} \\
& -\sum_{t=i}^{j} \gamma_{t}\left\langle\mathbb{E}\left[X_{t}-X^{*}\right], \mathbb{E}\left[e_{t}\left(\xi_{t}\right)\right]\right\rangle \\
\leq & d_{i}+\frac{1}{2}(M+N)^{2} \sum_{t=i}^{j} \gamma_{t}^{2} \\
& -\sum_{t=i}^{j} \gamma_{t}\left\langle\mathbb{E}\left[X_{t}-X^{*}\right], \mathbb{E}\left[e_{t}\left(\xi_{t}\right)\right]\right\rangle,
\end{array}
$$

and hence, setting $\nu_{t}=\gamma_{t} / \sum_{t=i}^{j} \gamma_{t}$, 


$$
\begin{aligned}
\mathbb{E}\left[\sum_{t=i}^{j} \nu_{t} f\left(X_{t}\right)-f\left(X^{*}\right)\right] & =\mathbb{E}\left[\sum_{t=i}^{j} \frac{\gamma_{t}}{\sum_{t=i}^{j} \gamma_{t}} f\left(X_{t}\right)-\sum_{t=i}^{j} \frac{\gamma_{t}}{\sum_{t=i}^{j} \gamma_{t}} f\left(X^{*}\right)\right]=\mathbb{E}\left[\sum_{t=i}^{j} \frac{\gamma_{t}}{\sum_{t=i}^{j} \gamma_{t}}\left[f\left(X_{t}\right)-f\left(X^{*}\right)\right]\right] \\
& =\frac{1}{\sum_{t=i}^{j} \gamma_{t}} \sum_{t=i}^{j} \gamma_{t} \mathbb{E}\left[f\left(X_{t}\right)-f\left(X^{*}\right)\right] \\
& \leq \frac{d_{i}+(1 / 2)(M+N)^{2} \sum_{t=i}^{j} \gamma_{t}^{2}-\sum_{t=i}^{j} \gamma_{t}\left\langle\mathbb{E}\left[X_{t}-X^{*}\right], \mathbb{E}\left[e_{t}\left(\xi_{t}\right)\right]\right\rangle}{\sum_{t=i}^{j} \gamma_{t}} .
\end{aligned}
$$

By convexity of $\Theta$, we have $\widetilde{X}_{i}^{j} \in X$, and by convexity of $f$, we have $f\left(\widetilde{X}_{i}^{j}\right) \leq \sum_{t=i}^{j} v_{t} f\left(X_{t}\right)$. Thus, by (43) and in view of $d_{1} \leq D_{\Theta}^{2}$ and $d_{i} \leq 4 D_{\Theta}^{2}$, $i>1$, we get

$$
\begin{aligned}
& \mathbb{E}\left[f\left(\widetilde{X}_{1}^{j}\right)-f\left(X^{*}\right)\right] \\
& \leq \frac{D_{\Theta}^{2}+(M+N)^{2} \sum_{t=i}^{j} \gamma_{t}^{2}+\sum_{t=i}^{j} \gamma_{t} D_{\Theta} N}{2 \sum_{t=i}^{j} \gamma_{t}}, \\
& \mathbb{E}\left[f\left(\widetilde{X}_{i}^{j}\right)-f\left(X^{*}\right)\right] \\
& \leq \frac{4 D_{\Theta}^{2}+(M+N)^{2} \sum_{t=i}^{j} \gamma_{t}^{2}+\sum_{t=i}^{j} \gamma_{t} D_{\Theta} N}{2 \sum_{t=i}^{j} \gamma_{t}} .
\end{aligned}
$$

Now we can develop step size policies along with the associated efficiency estimates based on (44). Assume that the number $n$ of iterations of the method is fixed in advance and that $\gamma_{t}=\gamma$. Then (44) becomes

$$
\begin{aligned}
& \mathbb{E}\left[f\left(\widetilde{X}_{1}^{j}\right)-f\left(X^{*}\right)\right] \\
& \leq \frac{D_{\Theta}^{2}+(M+N)^{2} n \gamma^{2}+n \gamma D_{\Theta} N}{2 n \gamma}, \\
& \mathbb{E}\left[f\left(\widetilde{X}_{i}^{j}\right)-f\left(X^{*}\right)\right] \\
& \leq \frac{4 D_{\Theta}^{2}+(M+N)^{2} n \gamma^{2}+n \gamma D_{\Theta} N}{2 n \gamma} .
\end{aligned}
$$

Minimizing the right-hand side of (45) over $\gamma>0$, we arrive at the constant step size policy

$$
\gamma=\frac{D_{\Theta}}{(M+N) \sqrt{n}}, \quad t=1, \ldots, N .
$$

Along with the associated efficiency estimate and $O\left(\mathbb{E}\left[e_{t}\left(\xi_{t}\right)\right]\right)<O(1 / N)$, we have

$$
\mathbb{E}\left[f\left(\widetilde{X}_{i}^{j}\right)-f\left(X^{*}\right)\right] \leq \frac{D_{\Theta}^{2}(5(M+N)+\sqrt{N})}{2 n \gamma} .
$$

With constant step size policy (47) in the above theorem, after $n$ iterations, the expected error is of order $O\left(n^{1 / 2}\right)$. This is worse than the rate for the inexact SA algorithm. However, the error bounds (48) are guaranteed independently of any smoothness and/or strong convexity assumptions on $f$.

\section{Specialization to the Case Where$$
\Theta=\{\mathscr{A}(X)=b, X \succeq 0\}
$$

To illustrate the advantage of inexact SA method over classical SA method, we apply our method to SCSDPs in this section. Problem (1) can be expressed in the form (8) with $\Theta=$ $\left\{\mathscr{A}(X)=b, X \geq 0, X \in S^{n}\right\}$. Subproblem (14) then becomes the following constrained minimization problem:

$$
\begin{array}{ll}
\min & \frac{1}{2}\left\|X-X_{j}+\gamma_{j} G\left(X_{j}, \xi_{j}\right)+\gamma_{j} e_{j}\right\|^{2} \\
\text { s.t. } & \mathscr{A}(X)=b, \\
& X \succeq 0 .
\end{array}
$$

The KKT corresponding to problem (49) is written as

$$
\begin{aligned}
& X_{j+1}-X_{j}+\gamma_{j} G\left(X_{j}, \xi_{j}\right)-\mathscr{A}^{*} \mu_{j+1}-\lambda_{j+1}+\gamma_{j} e_{j} \\
& \quad=0 \\
& \mathscr{A}\left(X_{j+1}\right)-b=0, \\
& \left\langle X_{j+1}, \lambda_{j+1}\right\rangle=\varepsilon_{j+1} \approx 0, \quad X_{j+1} \succeq 0, \lambda_{j+1} \succeq 0 .
\end{aligned}
$$

We can get the algorithm for problem (49).

Algorithm 10.

Step 1. Given the initial point $X_{1} \in \Theta$ and setting $j=1$, iterate the following steps.

Step 2. Choose suitable step sizes $\gamma_{j}>0$ and computational error $e_{j}$.

Step 3. Solve the following equation:

$$
\begin{aligned}
X_{j+1}-X_{j}+\gamma_{j} G\left(X_{j}, \xi_{j}\right)-\mathscr{A}^{*} \mu_{j+1}-\lambda_{j+1}+\gamma_{j} e_{j} & =0, \\
\mathscr{A}\left(X_{j+1}\right)-b & =0,
\end{aligned}
$$


TABLE 1: Objective function is random matrix.

\begin{tabular}{lcccc}
\hline & Radius & $r_{P}$ & $r_{D}$ & CPU time (s) \\
\hline$(1)$ & 0.5 & $5.4987 e-014$ & $3.8803 e-009$ & 0.2260 \\
$(2)$ & 1 & $5.1761 e-014$ & $9.8586 e-009$ & 0.2868 \\
$(3)$ & 1.5 & $3.4534 e-015$ & $5.4413 e-009$ & 0.5979 \\
$(4)$ & 2 & $7.9802 e-016$ & $1.8327 e-009$ & 0.7178 \\
$(5)$ & 2.5 & $4.5433 e-016$ & $6.1692 e-009$ & 0.8770 \\
$(6)$ & 3 & $8.3721 e-015$ & $3.4310 e-009$ & 0.9087 \\
$(7)$ & 3.5 & $2.6872 e-016$ & $3.1458 e-009$ & 1.1569 \\
$(8)$ & 4 & $4.5937 e-016$ & $9.1487 e-009$ & 1.2725 \\
$(9)$ & 4.5 & $8.6907 e-016$ & $8.1782 e-009$ & 1.3046 \\
$(10)$ & 5 & $6.6707 e-016$ & $6.1892 e-009$ & 1.3068 \\
$(11)$ & 6 & $5.6907 e-016$ & $3.1882 e-009$ & 1.5898 \\
\hline
\end{tabular}

$$
\begin{aligned}
\left\langle X_{j+1}, \lambda_{j+1}\right\rangle & =0, \\
X_{j+1} \geq 0, \quad \lambda_{j+1} & \geq 0 .
\end{aligned}
$$

Set $j:=j+1$; go back to Step 2 .

\section{Application and Numerical Results}

In order to assess from a practical point of view the inexact SA method, we code the Algorithm in MATLAB and ran it on several subcategories of SDP. We implement our algorithm in MATLAB 2009a and use a computer with one $2.20 \mathrm{GHz}$ processor and 2.00 GB RAM.

5.1. Numerical Results for Random Matrix. In our first example, we take a simple case of stochastic convex SDP

$$
\begin{array}{ll}
\min & f(X)=\|X-\mathbb{E}[A(\xi)]\|_{F} \\
\text { s.t. } & \operatorname{diag}(X)=e \\
& X \geq 0, X \in S^{4}
\end{array}
$$

where $f: S_{4}^{+} \times \Theta \rightarrow R$ is a convex function for every $\xi$ and

$$
A(\xi)=\left[\begin{array}{cccc}
2 & -1 & 0 & 0 \\
-1 & 2 & -1 & 0 \\
0 & -1 & 2 & -1 \\
0 & 0 & -1 & 2
\end{array}\right] \circ \xi
$$

where "o" means each element of $A$ multiplied by random variable $\xi$. We let $\xi$ mean 1 which is uniformly distributed whose interval radius is mutative from 0.5 to 4 . Using inexact SA method to solve this problem, we list the results in Table 1.

Table 1 shows that the inexact SA method for solving stochastic convex SDP is effective. The abscissa of Figure 1 represent the interval radius of $\xi$ and ordinate of Figure 1 represents norm difference of the optimal value between $\xi$ which is a random variable and $\xi \equiv 1$. This means that

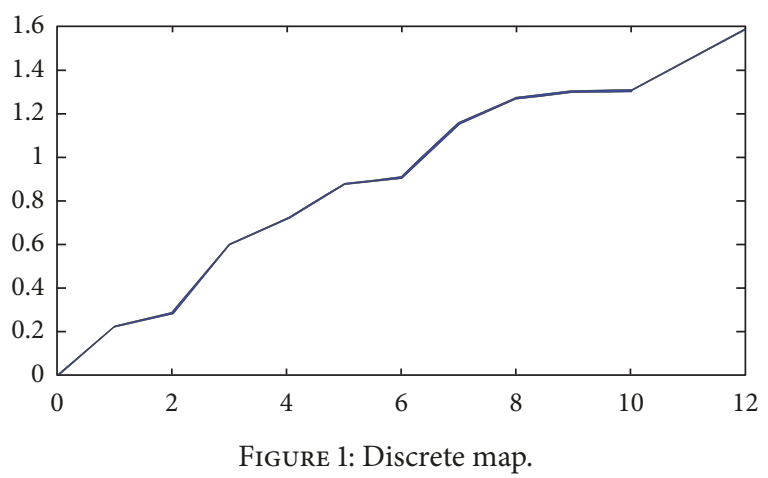

the variance of random variable may impact approximation; however, this influence does not exceed the tolerance range.

5.2. Numerical Results for SCQSDPs. In this example, we tested the inexact SA method on the linearly constrained SCQSDPs:

$$
\min \left\{\frac{1}{2} E[\langle x, \xi \mathscr{Q} x\rangle]+\langle c, x\rangle: \mathscr{A}(x)=b, x \geq 0\right\}
$$

where $\xi \sim U[0.5,1.5]$ is random variable, $\mathcal{Q}$, and $\mathscr{A}$ is given as follows:

$$
\begin{aligned}
& \mathscr{Q}(x)=\frac{1}{2}(B x+x B), \quad \text { where } B>0, \\
& \mathscr{A}(x)=\operatorname{diag}(x)=\left[x_{11}, x_{22}, \ldots, x_{n n}\right]^{T} .
\end{aligned}
$$

Then $b$ and $c$ can be generated at random. The MATLAB code for generating the matrix $b$ is given by $b=\operatorname{rand}(n, 1) ; b=$ $b$. $* b$. We generated $B$ as follows:

$$
\begin{aligned}
\text { randvec } & =1+9 * \operatorname{rand}(n, 1) \\
\operatorname{tmp} & =\operatorname{randn}\left(n, \text { ceil }\left(\frac{n}{4}\right)\right)
\end{aligned}
$$


TABLE 2: Objective function is stochastic quadratic.

\begin{tabular}{lcccccc}
\hline & $n$ & $r_{p}$ & $r_{D}$ & CPU time $(\mathrm{s})$ & Iter & Pobj \\
\hline$(1)$ & 100 & $9.2734 e-014$ & $1.0529 e-007$ & 17.46 & 18 & $6.8577 e+002$ \\
$(2)$ & 200 & $7.1942 e-014$ & $2.4666 e-007$ & 35.11 & 17 & $1.6196 e+003$ \\
$(3)$ & 300 & $5.2093 e-014$ & $5.1427 e-007$ & 90.36 & 16 & $2.3798 e+003$ \\
$(4)$ & 500 & $5.6795 e-014$ & $1.3859 e-007$ & 230.89 & 16 & $4.9247 e+003$ \\
$(5)$ & 800 & $4.5533 e-014$ & $1.1092 e-007$ & 756.32 & 20 & $8.5645 e+004$ \\
$(6)$ & 1000 & $4.3823 e-014$ & $7.4480 e-007$ & 1757.73 & 14 & $1.1458 e+004$ \\
$(7)$ & 1200 & $3.6972 e-014$ & $1.1656 e-007$ & 3939.16 & 17 & $4.9487 e+004$ \\
$(8)$ & 1500 & $3.6907 e-014$ & $1.1882 e-007$ & 7500.49 & 18 & $5.7514 e+005$ \\
\hline
\end{tabular}

$$
\begin{aligned}
& B=\operatorname{diag}(\text { randvec })+\frac{\left(\operatorname{tmp} * \mathrm{tmp}^{\prime}\right)}{n} ; \\
& B=\frac{\left(B+B^{\prime}\right)}{2} .
\end{aligned}
$$

We show the elementary numerical results in Table 2.

Our numerical results are reported in Table 2, where $n, r_{P}, r_{D}$, iter, and pobj stand for, respectively, the matrix dimension, the primal infeasibility, the dual infeasibility, the number of outer iterations, and the primal objective value. For each instance, our algorithm can solve the convex QSDP problem efficiently with very good primal infeasibility. Experimental result shows that the exact SA algorithm can achieve a high degree of detecting reliability.

5.3. Predict Correlation Coefficient in the Chinese Stock Market. In this example, we discuss how to use our inexact SA method to predict correlation coefficient in the Chinese stock market. This is the main motivation of this paper. Chinese stock market is different from other countries, and the rise or fall in a day is not more than ten percent. This feature could make us estimate some index more accurately.

In this paper, our main concern is the correlation coefficient of stocks. Correlation coefficient is often useful to know if two stocks tend to move together. For a diversified portfolio, you would want stocks that are not closely related. It helps to measure the closeness of the returns. Usually, we use the determinate correlation coefficient based on historical data. This principle which we mentioned in the previous paragraph makes us discover that we can use stochastic factor to estimate the correlation coefficient in the future.

According to the difference of the total market capitalization ( $=$ total equity $\times$ stock prices) of company, we can divide stocks into two parts called small-cap stocks and bigcap stocks.

(1) Small-Cap Stocks. They refer to stocks with a relatively small market capitalization. The definition of small cap can vary among brokerages, but generally it is a company with a market capitalization of less than $¥ 0.1$ billion.
Table 3 is the closing price of ten stocks of small-cap from 2017-6-3 to 2017-7-3, where CP is logogram of closing price. The second line of Table 3 is code of stocks.

We know that the change of the closing price in 2017.7.4 will not exceed ten percent and seriously up or down is small probability event. According to this and model (6), we can forecast the correlation coefficient from 2017.6.4 to 2017.7.4. We list the results in Table 4.

Now we give the true correlation coefficient of the stocks from 2017.6.4 to 2017.7.4 for comparison.

From Tables 4 and 5, we know that the correlation matrix we calculated is very close to the true correlation matrix. This mean that our method for predicting correlation matrix is effective.

(2) Big-Cap Stocks. In China, it is a term used by the investment community to refer to companies with a market capitalization value of more than $¥ 0.1$ billion. Large cap is an abbreviation of the term "large market capitalization." Market capitalization is calculated by multiplying the number of a company's shares outstanding by its stock price per share.

Table 6 is the closing price of ten stocks of big-cap from 2017-6-3 to 2017-7-3.

Like in the small-cap stocks, we calculate the correlation matrix as shown in Table 7.

Now we also give the true correlation coefficient of the stocks from 2017.6.4 to 2017.7.4 for comparison.

From Tables 7 and 8, we know that the correlation matrix we calculated is even more close to the true correlation matrix compared to the case in small-cap stocks. This means that our method for predicting correlation matrix is more effective in the company whose total market capitalization is huge.

\section{Conclusion}

In this paper, we propose stochastic convex semidefinite programs (SCSDPs) to handle data uncertainty in financial problem. An efficient inexact stochastic approximation method is designed. We proved the convergence, complexity, and robust treatment of the algorithm. Numerical experiments show that the method we proposed was effective for SCSDP and also for its special cases. We also numerically demonstrated that the method is more effective in big-cap stocks. 


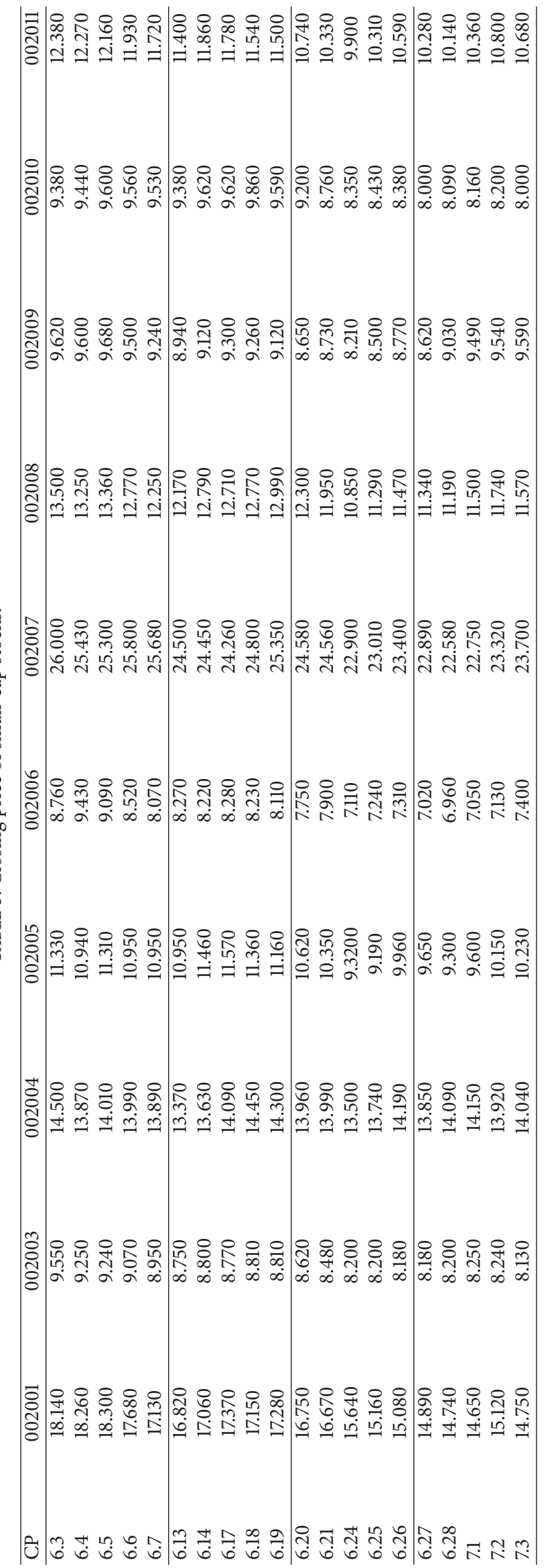


TABLe 4: Prediction correlation matrix.

\begin{tabular}{lcccccccccc}
\hline CC & 002001 & 002003 & 002004 & 002005 & 002006 & 002007 & 002008 & 002009 & 002010 & 002011 \\
\hline 002001 & 1 & 0.9597 & 0.0690 & 0.8547 & 0.9343 & 0.9042 & 0.9003 & 0.4002 & 0.9128 & 0.8682 \\
002003 & 0.9597 & 1 & 0.0681 & 0.8080 & 0.9129 & 0.9003 & 0.9110 & 0.6429 & 0.8139 & 0.9120 \\
002004 & 0.0690 & 0.0681 & 1 & 0.2535 & 0.1418 & 0.2719 & 0.2700 & 0.4429 & 0.1797 & 0.1379 \\
002005 & 0.8547 & 0.8080 & 0.2535 & 1 & 0.8080 & 0.8139 & 0.8896 & 0.5691 & 0.9100 & 0.8741 \\
002006 & 0.9343 & 0.9129 & 0.1418 & 0.8080 & 1 & 0.8517 & 0.8964 & 0.5837 & 0.8255 & 0.8888 \\
002007 & 0.9042 & 0.9003 & 0.2719 & 0.8139 & 0.8517 & 1 & 0.8624 & 0.5235 & 0.8381 & 0.8430 \\
002008 & 0.9003 & 0.9110 & 0.2700 & 0.8896 & 0.8964 & 0.8624 & 1 & 0.6828 & 0.8517 & 0.9168 \\
002009 & 0.4002 & 0.6429 & 0.4429 & 0.5691 & 0.5837 & 0.5235 & 0.6828 & 1 & 0.4691 & 0.7216 \\
002010 & 0.9128 & 0.8139 & 0.1797 & 0.9100 & 0.8255 & 0.8381 & 0.8517 & 0.4691 & 1 & 0.8430 \\
002011 & 0.8682 & 0.9120 & 0.1379 & 0.8741 & 0.8886 & 0.8430 & 0.9168 & 0.7216 & 0.8430 & 1 \\
\hline
\end{tabular}

TABle 5: True correlation matrix.

\begin{tabular}{lcccccccccc}
\hline CC & 002001 & 002003 & 002004 & 002005 & 002006 & 002007 & 002008 & 002009 & 002010 & 002011 \\
\hline 002001 & 1 & 0.966 & 0.016 & 0.852 & 0.948 & 0.905 & 0.917 & 0.342 & 0.928 & 0.871 \\
002003 & 0.966 & 1 & 0.043 & 0.833 & 0.957 & 0.908 & 0.931 & 0.493 & 0.908 & 0.929 \\
002004 & 0.016 & 0.043 & 1 & 0.172 & 0.016 & 0.133 & 0.236 & 0.388 & 0.106 & 0.09 \\
002005 & 0.852 & 0.833 & 0.172 & 1 & 0.821 & 0.832 & 0.916 & 0.512 & 0.913 & 0.899 \\
002006 & 0.948 & 0.957 & 0.016 & 0.821 & 1 & 0.868 & 0.923 & 0.496 & 0.848 & 0.911 \\
002007 & 0.905 & 0.908 & 0.133 & 0.832 & 0.868 & 1 & 0.87 & 0.437 & 0.864 & 0.849 \\
002008 & 0.917 & 0.931 & 0.236 & 0.916 & 0.923 & 0.87 & 1 & 0.583 & 0.898 & 0.935 \\
002009 & 0.342 & 0.493 & 0.388 & 0.512 & 0.496 & 0.437 & 0.583 & 1 & 0.339 & 0.647 \\
002010 & 0.928 & 0.908 & 0.106 & 0.913 & 0.848 & 0.864 & 0.898 & 0.339 & 1 & 0.875 \\
002011 & 0.871 & 0.929 & 0.09 & 0.899 & 0.911 & 0.849 & 0.935 & 0.647 & 0.875 & 1 \\
\hline
\end{tabular}

TABLE 6: Closing price of big-cap stocks.

\begin{tabular}{lcccccccccc}
\hline CP & 002001 & 002003 & 002004 & 002005 & 002006 & 002007 & 002008 & 002009 & 002010 & 002011 \\
\hline 6.3 & 9.840 & 4.790 & 10.71 & 10.38 & 4.81 & 6.740 & 12.990 & 9.280 & 13.770 & 12.350 \\
6.4 & 9.740 & 4.730 & 10.70 & 10.28 & 4.77 & 6.730 & 12.740 & 9.160 & 13.750 & 12.180 \\
6.5 & 9.650 & 4.770 & 10.59 & 10.10 & 4.76 & 6.710 & 12.880 & 9.060 & 13.360 & 12.150 \\
6.6 & 9.450 & 4.700 & 10.39 & 10.00 & 4.71 & 6.710 & 12.720 & 9.040 & 13.180 & 11.760 \\
6.7 & 9.350 & 4.560 & 10.26 & 9.990 & 4.66 & 6.600 & 12.120 & 8.840 & 13.170 & 11.520 \\
\hline 6.13 & 9.020 & 4.300 & 9.990 & 9.880 & 4.53 & 6.390 & 11.350 & 8.340 & 12.320 & 11.000 \\
6.14 & 9.020 & 4.500 & 10.06 & 9.960 & 4.55 & 6.290 & 11.340 & 8.310 & 12.210 & 11.070 \\
6.17 & 9.010 & 4.620 & 10.03 & 9.910 & 4.51 & 6.350 & 11.120 & 8.160 & 12.100 & 10.970 \\
6.18 & 9.070 & 4.590 & 10.10 & 9.950 & 4.33 & 6.350 & 11.220 & 8.140 & 12.200 & 11.120 \\
6.19 & 8.890 & 4.560 & 9.960 & 9.750 & 4.28 & 4.680 & 11.150 & 8.110 & 11.980 & 10.940 \\
\hline 6.20 & 8.420 & 4.340 & 9.520 & 9.290 & 4.17 & 4.500 & 10.820 & 7.940 & 11.470 & 10.610 \\
6.21 & 8.280 & 4.370 & 9.480 & 9.450 & 4.15 & 4.450 & 11.050 & 7.730 & 11.710 & 10.590 \\
6.24 & 7.520 & 4.060 & 8.690 & 8.510 & 4.05 & 4.210 & 10.090 & 7.480 & 10.930 & 9.5300 \\
6.25 & 7.800 & 3.960 & 8.690 & 8.440 & 4.03 & 4.190 & 10.000 & 7.540 & 10.920 & 9.3000 \\
6.26 & 7.770 & 3.930 & 8.580 & 8.300 & 3.92 & 4.100 & 9.9900 & 7.490 & 10.660 & 9.3100 \\
\hline 6.27 & 7.880 & 4.230 & 8.660 & 8.210 & 3.92 & 4.110 & 9.8400 & 7.450 & 10.920 & 9.3300 \\
6.28 & 8.280 & 4.100 & 9.020 & 8.570 & 3.93 & 4.180 & 10.130 & 7.510 & 11.600 & 9.9100 \\
7.1 & 8.170 & 4.030 & 8.920 & 8.590 & 3.91 & 4.270 & 10.180 & 7.420 & 11.380 & 9.9900 \\
7.2 & 8.150 & 3.990 & 8.880 & 8.480 & 3.92 & 4.240 & 10.190 & 7.280 & 11.210 & 9.8700 \\
7.3 & 8.040 & 3.940 & 8.680 & 8.440 & 3.94 & 4.250 & 9.9700 & 7.060 & 11.350 & 9.6600 \\
\hline
\end{tabular}


TABLE 7: Prediction correlation matrix.

\begin{tabular}{lcccccccccc}
\hline CC & 600000 & 600010 & 600015 & 600016 & 600019 & 600028 & 600030 & 600031 & 600036 & 600048 \\
\hline 600000 & 1 & 0.9119 & 0.9776 & 0.9497 & 0.9388 & 0.9656 & 0.9447 & 0.9427 & 0.9766 & 0.9736 \\
600010 & 0.9119 & 1 & 0.9477 & 0.9318 & 0.9209 & 0.9298 & 0.9020 & 0.8960 & 0.9030 & 0.9318 \\
600015 & 0.9776 & 0.9477 & 1 & 0.9825 & 0.9646 & 0.9835 & 0.9467 & 0.9408 & 0.9716 & 0.9825 \\
600016 & 0.9497 & 0.9318 & 0.9825 & 1 & 0.9437 & 0.9666 & 0.9050 & 0.8940 & 0.9547 & 0.9597 \\
600019 & 0.9388 & 0.9209 & 0.9646 & 0.9437 & 1 & 0.9766 & 0.9587 & 0.9716 & 0.9358 & 0.9527 \\
600028 & 0.9656 & 0.9298 & 0.9835 & 0.9666 & 0.9766 & 1 & 0.9597 & 0.9557 & 0.9567 & 0.9756 \\
600030 & 0.9447 & 0.9020 & 0.9467 & 0.9050 & 0.9587 & 0.9597 & 1 & 0.9776 & 0.9408 & 0.9696 \\
600031 & 0.9427 & 0.8960 & 0.9408 & 0.8940 & 0.9716 & 0.9557 & 0.9776 & 1 & 0.9318 & 0.9487 \\
600036 & 0.9766 & 0.9030 & 0.9716 & 0.9547 & 0.9358 & 0.9567 & 0.9408 & 0.9318 & 1 & 0.9746 \\
600048 & 0.9736 & 0.9318 & 0.9825 & 0.9597 & 0.9527 & 0.9756 & 0.9696 & 0.9487 & 0.9746 & 1 \\
\hline
\end{tabular}

TABLE 8: True correlation matrix.

\begin{tabular}{lcccccccccc}
\hline CC & 600000 & 600010 & 600015 & 600016 & 600019 & 600028 & 600030 & 600031 & 600036 & 600048 \\
\hline 600000 & 1 & 0.911 & 0.979 & 0.952 & 0.937 & 0.97 & 0.943 & 0.932 & 0.979 & 0.976 \\
600010 & 0.911 & 1 & 0.951 & 0.932 & 0.92 & 0.925 & 0.901 & 0.9 & 0.887 & 0.93 \\
600015 & 0.979 & 0.951 & 1 & 0.987 & 0.968 & 0.985 & 0.951 & 0.944 & 0.968 & 0.988 \\
600016 & 0.952 & 0.932 & 0.987 & 1 & 0.946 & 0.97 & 0.907 & 0.893 & 0.955 & 0.965 \\
600019 & 0.937 & 0.92 & 0.968 & 0.946 & 1 & 0.981 & 0.962 & 0.972 & 0.929 & 0.954 \\
600028 & 0.97 & 0.925 & 0.985 & 0.97 & 0.981 & 1 & 0.965 & 0.952 & 0.96 & 0.982 \\
600030 & 0.943 & 0.901 & 0.951 & 0.907 & 0.962 & 0.965 & 1 & 0.975 & 0.934 & 0.973 \\
600031 & 0.932 & 0.9 & 0.944 & 0.893 & 0.972 & 0.952 & 0.975 & 1 & 0.909 & 0.942 \\
600036 & 0.979 & 0.887 & 0.968 & 0.955 & 0.929 & 0.96 & 0.934 & 0.909 & 1 & 0.973 \\
600048 & 0.976 & 0.93 & 0.988 & 0.965 & 0.954 & 0.982 & 0.973 & 0.942 & 0.973 & 1 \\
\hline
\end{tabular}

\section{Conflicts of Interest}

The authors declare that there are no conflicts of interest regarding the publication of this paper.

\section{Acknowledgments}

The work is partially supported by the Natural Science Foundation of China, Grants 11626051, 11626052, 11701061, and 11501074.

\section{References}

[1] K. Ariyawansa and Y. Zhu, "A class of polynomial volumetric barrier decomposition algorithms for stochastic semidefinite programming," Mathematics of Computation, vol. 80, no. 275, pp. 1639-1661, 2011.

[2] K. A. Ariyawansa and Y. Zhu, "Stochastic semidefinite programming: a new paradigm for stochastic optimization," $4 O R$, vol. 4 , no. 3, pp. 239-253, 2006.

[3] S. Jin, K. A. Ariyawansa, and Y. Zhu, "Homogeneous self-dual algorithms for stochastic semidefinite programming," Journal of Optimization Theory and Applications, vol. 155, no. 3, pp. 10731083, 2012.

[4] S. Mehrotra and M. G. Gozevin, "Decomposition-based interior point methods for two-stage stochastic semidefinite programming," SIAM Journal on Optimization, vol. 18, no. 1, pp. 206-222, 2007.

[5] Y. Zhu, J. Zhang, and K. Partel, "Location-aided routing with uncertainty in mobile ad hoc networks: A stochastic semidefinite programming approach," Mathematical and Computer Modelling, vol. 53, no. 11-12, pp. 2192-2203, 2011.

[6] N. J. Higham, "Computing the nearest correlation matrixa problem from finance," IMA Journal of Numerical Analysis (IMAJNA), vol. 22, no. 3, pp. 329-343, 2002.

[7] F. Alizadeh, "Interior point methods in semidefinite programming with applications to combinatorial optimization," SIAM Journal on Optimization, vol. 5, no. 1, pp. 13-51, 1995.

[8] K. Jiang, D. Sun, and K. C. Toh, "An inexact accelerated proximal gradient method for large scale linearly constrained convex SDP," SIAM Journal on Optimization, vol. 22, no. 3, pp. 1042-1064, 2012.

[9] M. J. Todd, "Semidefinite optimization," Acta Numerica, vol. 10, no. 126, pp. 515-560, 2001.

[10] L. Vandenberghe and S. Boyd, "Semidefinite programming," SIAM Review, vol. 38, no. 1, pp. 49-95, 1996.

[11] H. Wolkowicz, R. Saigal, and L. Vandenberghe, Handbook of semidefinite programming: theory, algorithms, and applications, vol. 27, Kluwer Academic Pub, New York, NY, USA, 2000.

[12] J. Malick, "A dual approach to semidefinite least-squares problems," SIAM Journal on Matrix Analysis and Applications, vol. 26, no. 1, pp. 272-284, 2004.

[13] H. Qi and D. Sun, "A quadratically convergent Newton method for computing the nearest correlation matrix," SIAM Journal on Matrix Analysis and Applications, vol. 28, no. 2, pp. 360-385, 2006.

[14] K. C. Toh, R. H. Tutuncu, and M. J. Todd, "Inexact primal-dual path-following algorithms for a special class of convex quadratic SDP and related problems," Pacific Journal of Optimization, vol. 3, pp. 135-164, 2007. 
[15] S. Ghadimi and G. Lan, "Stochastic first- and zeroth-order methods for nonconvex stochastic programming," Siam Journal on Optimization, vol. 23, no. 4, pp. 2341-2368, 2013.

[16] G. Lan, A. Nemirovski, and A. Shapiro, "Validation analysis of mirror descent stochastic approximation method," Mathematical Programming, vol. 134, no. 2, pp. 425-458, 2012.

[17] G. Lan and S. Ghadimi, "Optimal stochastic approximation algorithms for strongly convex stochastic composite optimization i: a generic algorithmic framework," Siam Journal on Optimization, vol. 22, no. 4, pp. 1469-1492, 2012.

[18] G. Lan and S. Ghadimi, "Optimal stochastic approximation algorithms for strongly convex stochastic composite optimization, ii: shrinking procedures and optimal algorithms," Siam Journal on Optimization, vol. 23, no. 4, pp. 2061-2089, 2010.

[19] G. Lan, "An optimal method for stochastic composite optimization," Mathematical Programming, vol. 133, no. 1-2, Ser. A, pp. 365-397, 2012.

[20] A. Nemirovski and D. Yudin, "On Cezaris convergence of the steepest descent method for approximating saddle point of convex-concave functions," in Soviet Math. Dokl, vol. 19, 1978.

[21] A. Nemirovski, A. Juditsky, G. Lan, and A. Shapiro, "Robust stochastic approximation approach to stochastic programming," SIAM Journal on Optimization, vol. 19, no. 4, pp. 15741609, 2008.

[22] H. Robbins and D. Siegmund, "A convergence theorem for nonnegative almost supermartingales and some applications," in J. S. Rustagi, Optimizing Methods in Statistics, pp. 233-257, Academic Press, New York, NY, USA, 1971.

[23] A. S. Nemirovsky and D. B. Yudin, 1983, Problem complexity and method efficiency in optimization. 


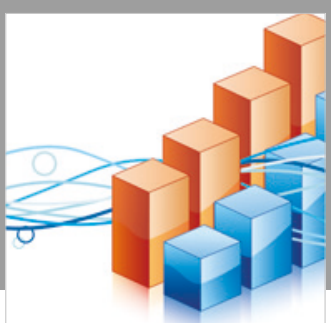

Advances in

Operations Research

\section{-n-m}
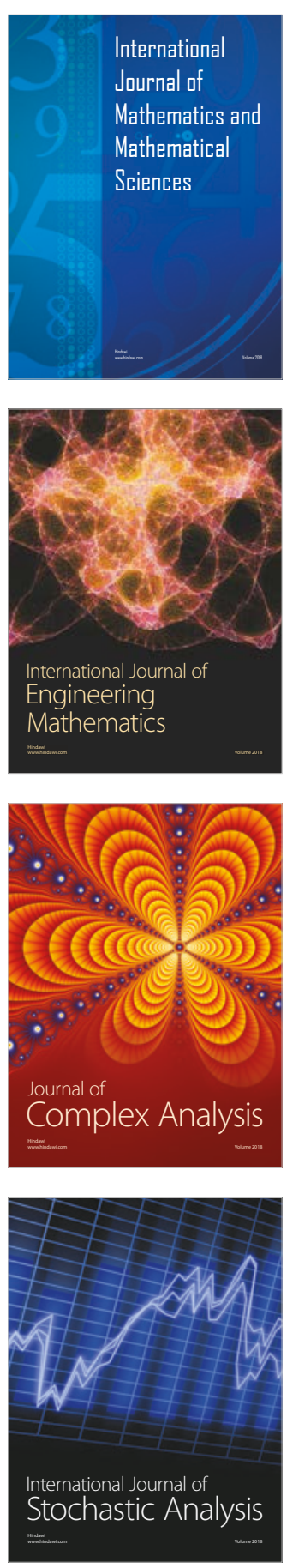
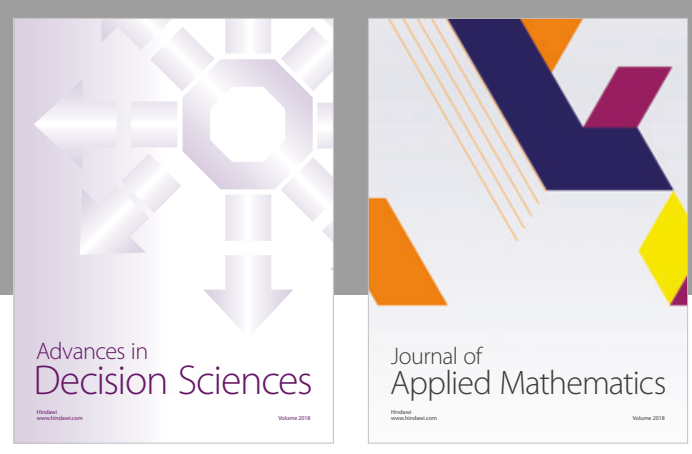

Journal of

Applied Mathematics
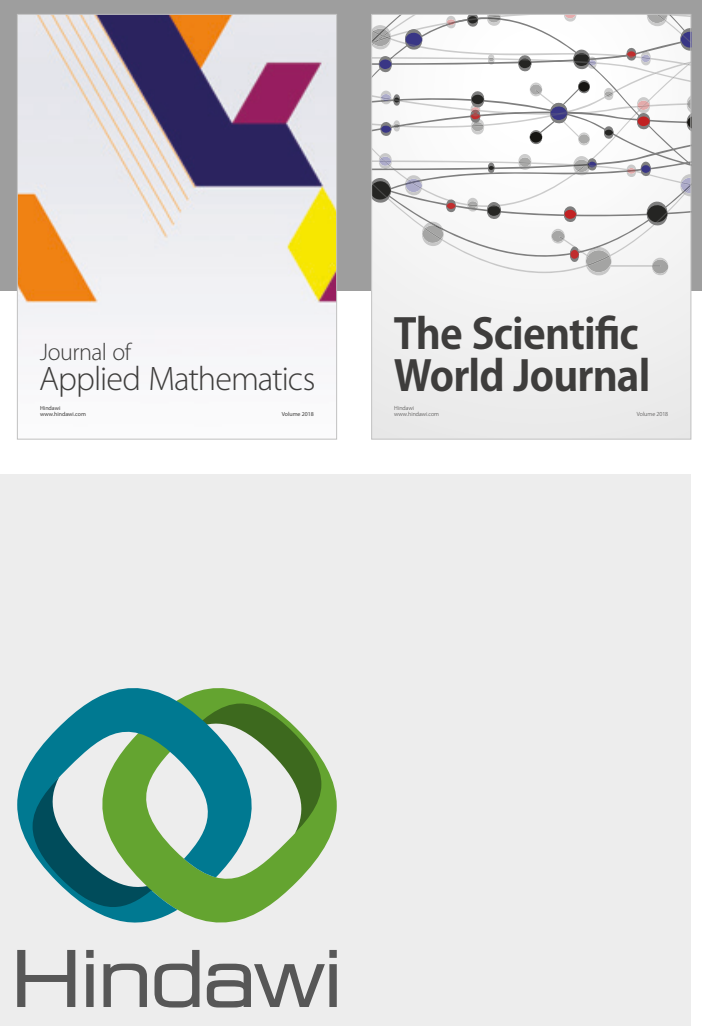

Submit your manuscripts at

www.hindawi.com

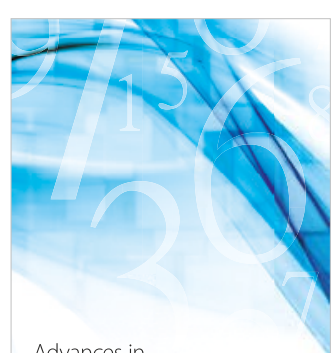

Advances in
Numerical Analysis
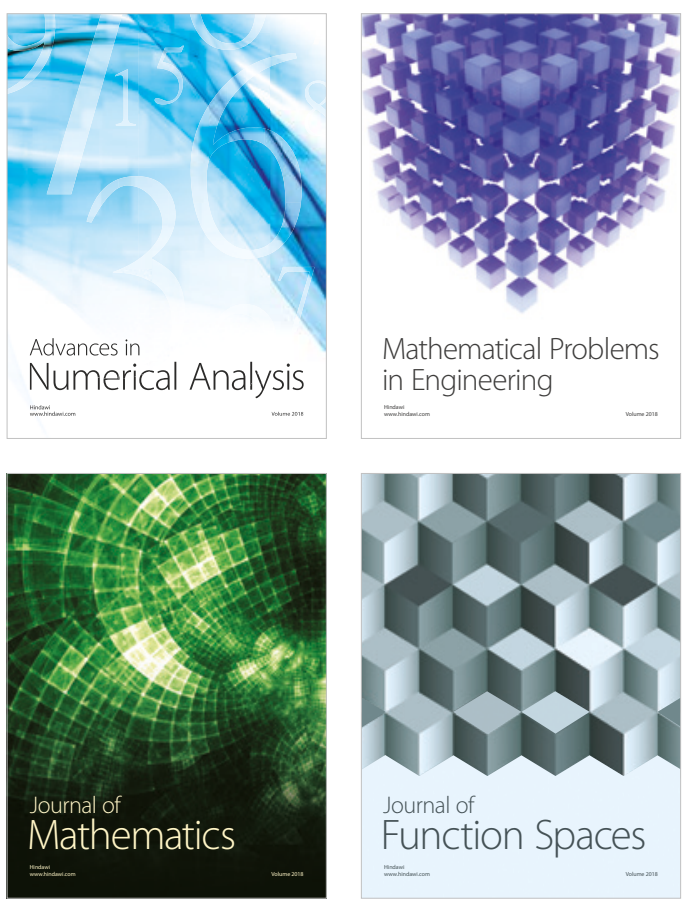

Mathematical Problems in Engineering

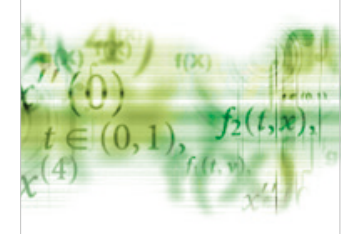

International Journal of

Differential Equations

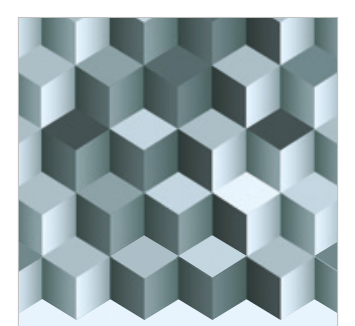

Journal of

Function Spaces

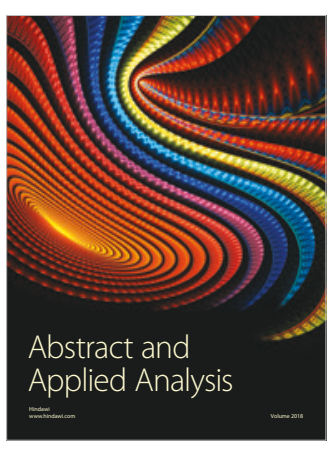

The Scientific

World Journal

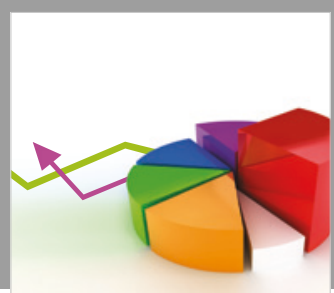

Journal of

Probability and Statistics
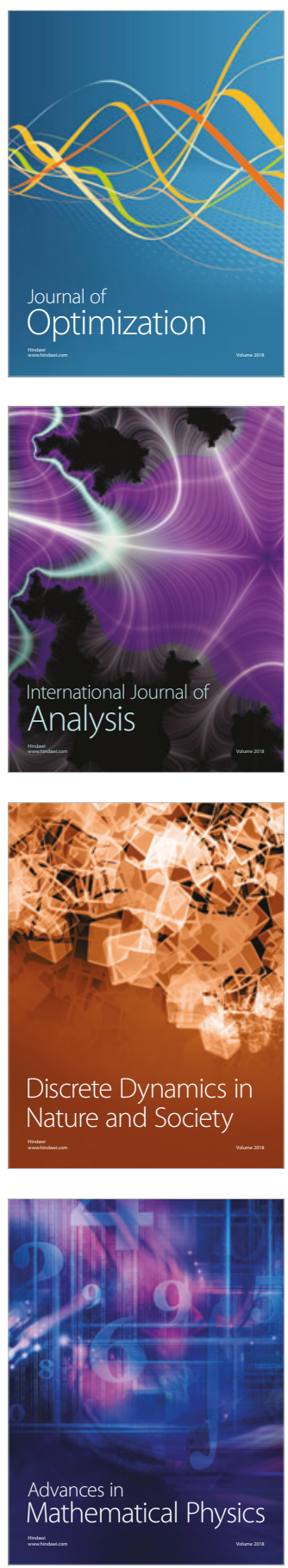